\title{
Empiema subdural como complicación de sinusitis
}

\author{
Raúl O Bustos B, Paula A Pavéz M, Bernardo J Bancalari M, \\ Rubén M Miranda Ay Héctor R Escobar S.
}

\section{Subdural empyema secondary to sinusitis}

Subdural empyema is a rare complication of sinusitis in children. Its clinical presentation represents a neurosurgical emergency and as a scarcely recognized entity a delayed diagnosis rapidly increases its fatal prognosis. We report the clinical and radiological course of an adolescent with a subdural empyema secondary to sinusitis. Clinical and radiological features, laboratory findings and outcome of this condition are discussed based in a review of previously reported cases.

Key words: sinusitis, subdural abscess, pediatrics.

Palabras claves: sinusitis, absceso subdural, pediatría.

\section{Introducción}

$\mathrm{H}$ oy en día, el empiema subdural (ESD), definido como una colección purulenta en el espacio potencial que se sitúa entre la capa interna de la duramadre y la membrana externa de la aracnoides, constituye una condición poco frecuente en pediatría y se acompaña de una elevada morbimortalidad si no es diagnosticado precozmente.

En décadas pasadas, aproximadamente $25 \%$ de las infecciones bacterianas intracraneanas en niños correspondían a un ESD, siendo la mayoría de estos casos secundarios a meningitis bacteriana aguda (MBA). La inmunización contra Haemophilus influenzae tipo b ha hecho disminuir la incidencia del ESD en edades más precoces, siendo actualmente más común su presentación en adolescentes y adultos, donde su origen es principalmente secundario a infecciones de los senos paranasales ${ }^{1}$. Un particular peligro de esta infección radica en que la anatomía del espacio subdural permite una distribución uniforme del proceso infeccioso en ambos hemiferios cerebrales, los que se comprometen en cerca de $70 \%$ de los casos.

En la era pre-antimicrobiana la mortalidad de esta condición era cercana al $100 \%$; sin embargo, en las últimas décadas, el diagnóstico precoz con neuroimagenología, el uso de antimicrobianos de amplio espectro y la evacuación quirúrgica han disminuido notablemente su letalidad.

Esta comunicación tiene como objetivo describir la presentación clínica, evolución y tratamiento de un escolar previamente sano que cursó con un empiema subdural secundario a una sinusitis. Basados en una búsqueda realizada en la base de datos Scientific Electronic Library Online en Chile (www.SCIELO.cl), a partir del año 1998, a nuestro entender esta condición no ha sido descrita previamente en la literatura médica nacional.

\section{Caso clínico}

Escolar de sexo masculino, con 10 años 9 meses de edad, sin antecedentes mórbidos de importancia. Presentaba historia de una semana de evolución de cefalea, fiebre y edema periorbitario derecho, por lo que se hospitalizó en otro centro donde se le realizó una TAC de senos paranasales y de órbita que demostró una pansinusitis y sospecha de absceso orbitario derecho iniciándose tratamiento antimicrobiano con ceftriaxona y cloxacilina (Figura 1). A las 48 horas del ingreso, el paciente cursó con un cuadro confusional agudo y crisis de agitación psicomotora asociado a signos de irritación meníngea, por lo que se le realizó una punción lumbar obteniéndose LCR compatible con una meningitis (490 células $/ \mathrm{mm}^{3} \quad 60 \% \mathrm{PMN}$, glucorraquia y proteínas normales), pero el cultivo del LCR y sangre fueron negativos. Una TAC de cerebro con medio de contraste evidenció edema cerebral y una colección subdural bilateral por lo que se trasladó a nuestro centro para evaluación neuro-quirúrgica con los diagnósticos de pansinusitis, meningitis aguda secundaria y colección subdural.

Al ingreso a la unidad de cuidados intensivos
Universidad de Concepción: Facultad de Medicina, Departamento de Pediatría (RBB, PAPM) Hospital Guillermo Grant Benavente, Concepción: Unidad de Cuidado Intensivo Pediátrico (RBB, BBM, RMA, HES)

Recibido: 02-08-05 Aceptado: 12-12-05

Correspondencia a: Raúl O Bustos B rabustos@udec.cl 


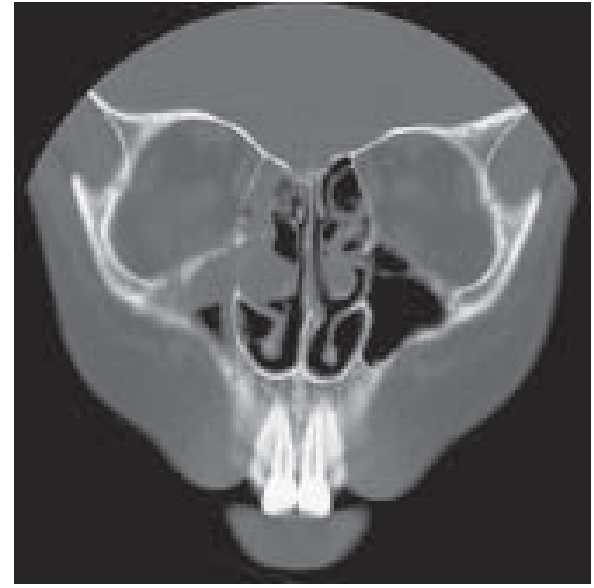

Figura 1. Tomografía axial computada de senos paranasales inicial. Ocupación de compartimientos etmoidales y seno maxilar derecho con rarefacción ósea en la lámina cribosa etmoidal y participación de tejidos blandos orbitarios.

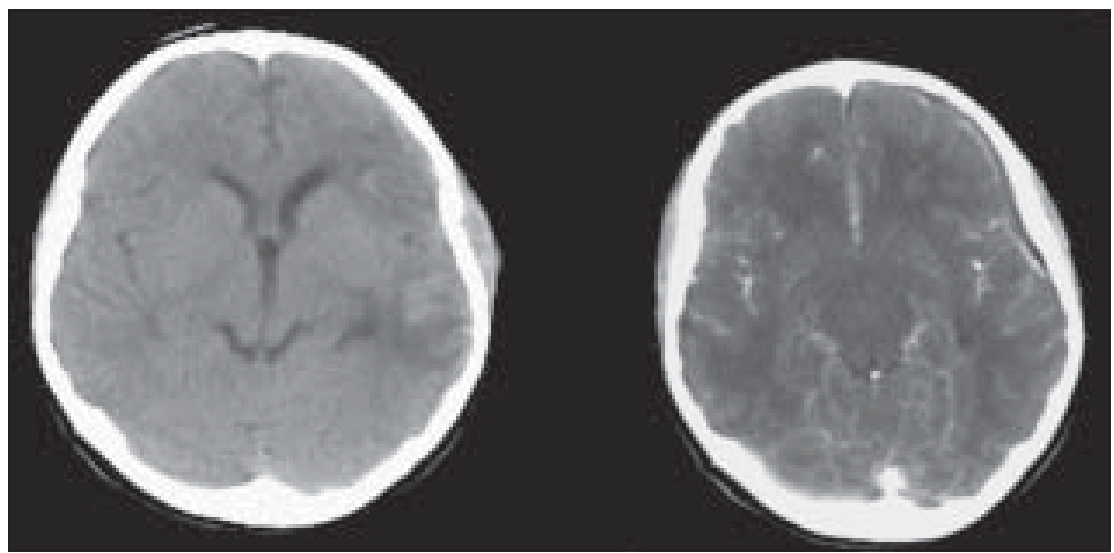

Figura 2. Tomografía axial computada de cerebro sin medio de contraste. (Figura 2a): Se aprecia asimetría de las cisuras y circunvoluciones con desplazamiento de la línea media que sugiere efecto de ocupación a derecha. Tomografía axial computada de cerebro con medio de contraste (Figura 2b). Empiema subdural izquierdo.

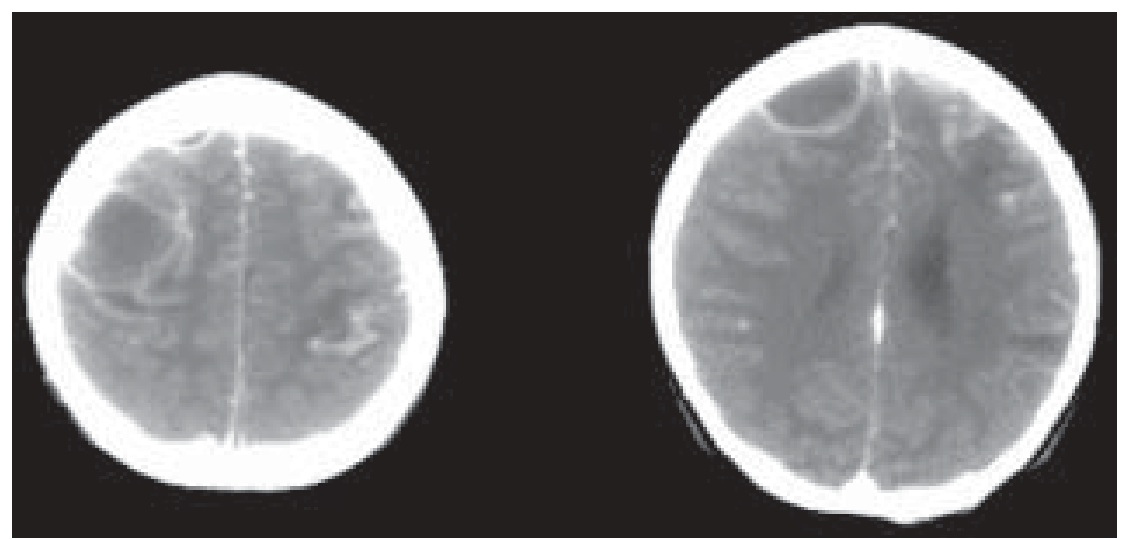

Figura 3. Tomografía axial computada de cerebro con medio de contraste. Lesión hipodensa proyectada en el lóbulo parietofrontal y frontal derechos con realce periférico sugerente de colección. pediátricos destacaba al examen físico: $\mathrm{T}^{\circ}$ axilar $38,3{ }^{\circ} \mathrm{C}$, frecuencia cardíaca de 65 por min, presión arterial 140/ $90 \mathrm{~mm} \mathrm{Hg}$, escala de Glasgow: 6, hiperreflexia, anisocoria midriática a derecha, rigidez de nuca, edema bipalpebral y quemosis, clonus bilateral mayor a derecha. La PCR fue de $215 \mathrm{mg} / \mathrm{L}$, el recuento leucocitario de $15.800 / \mathrm{mm}^{3}$, y el recuento de plaquetas normal. Se inició manejo de la hipertensión endocraneana con intubación endotraqueal y conexión a ventilación mecánica asociada a la administración de manitol, sedoanalgesia con midazolam-fentanilo y soporte inotrópico con dopamina. Se instauró terapia anticonvulsivante con fenitoína y tratamiento antimicrobiano triasociado con ceftriaxona, metronidazol y vancomicina. Una nueva TAC cerebral con y sin contraste mostró edema cerebral y progresión de la colección subdural planteándose el diagnóstico de empiema subdural con efecto de masa inicial (Figura 2). El paciente fue evaluado por el equipo de otorrinolaringología y neurocirugía realizándose exploración del meato medio y drenaje del seno maxilar derecho por vía endoscópica y en las horas siguientes etmoidectomía derecha, drenaje de un absceso orbitario derecho y drenaje de un empiema subdural izquierdo desde donde se retiró abundante material purulento. El cultivo microbiológico de estas zonas para aerobios y anaerobios estrictos, procesado en sistema BACTEC/ALERT® ${ }^{\circledR}$ FN con bajo potencial de oxido reducción y carbón activado, fue negativo.

El paciente permaneció en ventilación asistida durante siete días y cursó con regresión de los signos de hipertensión endocraneana y recuperación progresiva

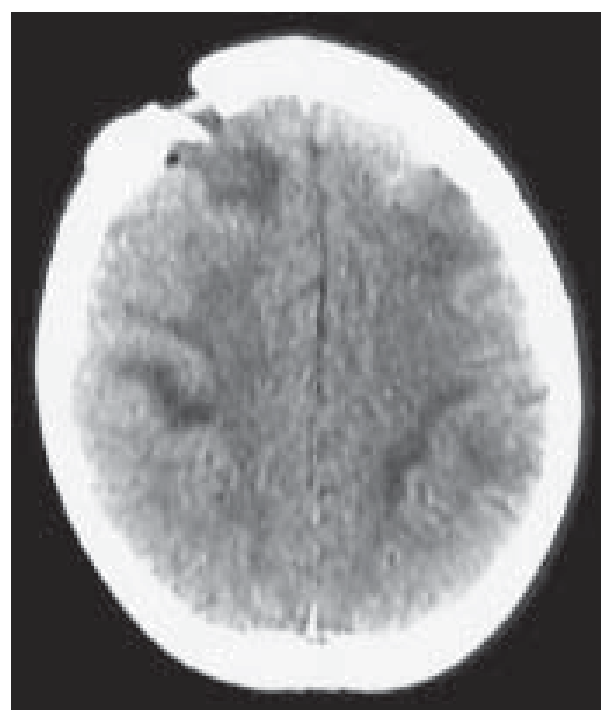

Figura 4. Tomografía axial computada de cerebro con medio de contraste, posterior a craneotomia frontal derecha. 
en la escala de Glasgow. Tras tres semanas de antibioterapia triasociada, se realizó nueva TAC cerebral con medio de contraste que mostró dos colecciones encefálicas frontales derechas (Figura 3), por lo que debió realizarse una nueva intervención quirúrgica: cranectomía con vaciamiento frontal derecho, drenaje de empiema con dos agujeros de trépano y durotomía que dio salida a pus abundante. Los cultivos nuevamente resultaron negativos.

La evolución posterior fue favorable, completando 5 semanas de antibioterapia y el seguimiento con TAC cerebral con medio de contraste no reveló la formación de nuevas colecciones (Figura 4). En los controles clínicos post egreso el paciente presentaba una afasia de expresión, logrando comunicación en forma escrita y una hemiparesia derecha actualmente en regresión.

\section{Discusión}

Actualmente, en la mayoría de las series, el ESD secundario a sinusitis es una enfermedad del período escolar y adolescente, con un predominio del género masculino en alrededor de $60 \%$ de los casos. El rápido crecimiento y gran tamaño de los senos frontales, asociado al desarrollo de una abundante vascularización en este período de la vida, explicarían la mayor incidencia de ESD en este grupo etario. Entre las causas de ESD, la sinusitis aparece como el factor inicial en $\sim 70 \%$ de los casos, siendo el seno frontal el más frecuentemente involucrado, la otitis media contribuye con $\sim 20 \%$ de los casos y en pacientes bajo 5 años de edad, la MBA es una causa importante en algunas zonas donde no hay programas de vacunación contra Haemophilus influenzae tipo $\mathrm{b}^{2}$. También se han descrito ESD secundarios a lesiones traumáticas y post craneotomía.

En la patogénesis del ESD secundario a una sinusitis se han descrito dos vías de propagación, la directa que implica la erosión de la pared posterior del seno frontal, llegando así a la duramadre, y una vía indirecta que se produce por tromboflebitis retrógrada de las venas de la mucosa de los senos paranasales, siendo este último mecanismo el más frecuente ${ }^{3}$.

La presentación clínica en escolares y adolescentes incluye fiebre, cefalea, irritabilidad náuseas, vómitos y como en nuestro paciente, irritación meningea, celulitis periorbitaria con proptosis ocular y alteración de la conciencia. En general esta sintomatología puede tener una duración de una a dos semanas previa a la confirmación diagnóstica. El médico clínico debería sospechar un empiema subdural frente a un paciente febril con una sinusitis y la aparición posterior de síntomas neurológicos.
La ruta de infección va a determinar la etiología del ESD, de tal manera que, como gran parte de los casos se originan en los senos paranasales, la mayoría de los microorganismos involucrados son Streptococcus aerobios y anaerobios estrictos (principalmente de la especie Streptococcus intermedius) los cuales son aislados hasta en $60 \%$ de los casos. Las infecciones postoperatorias o postraumáticas son generalmente secundarias a Staphylococcus aureus y Staphylococcus coagulasa negativa y ocasionalmente bacilos gramnegativos ${ }^{4-5}$. Streptococcus pneumoniae, a pesar de ser un agente frecuente de sinusitis en niños, tiene escasa representación como agente etiológico de ESD $(2 \%)^{4}$.

La punción lumbar, que se realizó en este caso, está formalmente contraindicada en los pacientes con ESD: por un lado, su aporte al diagnóstico microbiológico es pobre, ya que en la mayoría de los casos no se logra identificar bacterias en el LCR y además es potencialmente letal en pacientes con signos de aumento de la presión intracraneana, pudiendo producir una herniación cerebral. En un tercio de los casos de ESD el estudio etiológico realizado al exudado presente en el espacio subdural es negativo, probablemente debido al uso previo de antimicrobianos y la falta de toma de cultivos para microorganismos anaerobios estrictos de manera rutinaria ${ }^{6}$.

En conjunto con la clínica, el diagnóstico del ESD se realiza por neuroimágenes. Actualmente, la resonancia magnética (RM) con gadolinium ha pasado a ser el examen de elección. La RM tiene mejor sensibilidad que la TAC para la detección de pequeñas colecciones líquidas, es más específica en la diferenciación entre empiema y efusión y permite definir con más exactitud los límites anatómicos de la infección y mejor visualización de la fosa posterior? ${ }^{7}$.

El tratamiento del ESD es médico-quirúrgico. El manejo médico implica el control de la hipertensión endocraneana secundaria al edema cerebral que por efecto de masa ha provocado el ESD, y el uso de antimicrobianos sistémicos de amplio espectro cuya elección dependerá del origen del ESD. Así, en los casos secundarios a sinusitis, en la literatura extranjera se recomienda una terapia triasociada con vancomicina, metronidazol y una cefalosporina de tercera generación No obstante, en nuestro medio, en los casos secundarios a otitis o sinusitis, el uso de cefalosporinas de tercera generación sería adecuada ya que se ha demostrado buena actividad in vitro, con una susceptibilidad de $98 \%$ para Streptococcus del grupo anginos $^{8}{ }^{8}$, asociado a agentes con cobertura anaerobia y debiera reservarse el uso de vancomicina para ESD secundarios a cirugías o traumas. Respecto a la duración óptima del tratamiento antiinfeccioso no exis- 
ten guías prestablecidas, pero en general distintas series reportan su uso durante 3 a 6 semanas por vía intravenosa; un plazo que aparece razonable es continuar el tratamiento antimicrobiano por 3 a 4 semanas después del drenaje quirúrgico y extender su administración si la condición clínica o radiológica del paciente no mejora.

Concomitante al manejo médico, el tratamiento quirúrgico consiste en la evacuación de material purulento ya que disminuye el efecto de masa, permite una mejor penetración de los antimicrobianos y podría llevar a la identificación y susceptibilidad de los agentes responsables. La craneotomía o la trepanación han sido utilizadas en el tratamiento quirúrgico del ESD. Algunos autores recomiendan la trepanación en aquellos pacientes críticamente enfermos, donde la craneotomía se acompaña de gran riesgo, en las fases precoces de la enfermedad, cuando existe pus de poca viscosidad o en aquellas lesiones supratentoriales. La craneotomía sería más apropiada en lesiones infratentoriales, difusas y multiloculadas. Por otro lado, esta segunda técnica ofrece la oportunidad de inspeccionar el sitio de infección, remover tejido necrótico y bolsillos loculados de pus, accediendo a áreas donde la trepanación no llega. También la literatura médica hace énfasis en que un enfrentamiento quirúrgico multidisciplinario (otorrinolaringológico y neuroquirúrgico) de esta infección llevaría a una disminución en la necesidad de reexploración'. Como en nuestro caso, a pesar de un tratamiento médico y quirúrgico agresivo, en algunas series hasta un tercio de los pacientes van a requerir múltiples cirugías para evacuar colecciones persistentes o recurrentes ${ }^{10}$.

La letalidad de esta condición oscila entre 6 y $17 \%$ siendo las causas postraumáticas las que se asocian a mayor mortalidad. La morbilidad expresada como déficit neurológico persistente, hemiparesia o convulsiones, puede llegar hasta $40 \%$ de los $\operatorname{casos}^{11}$.

En conclusión, se presenta un caso de empiema subdural secundario a sinusitis en que el manejo multidisciplinario (cuidados intensivos, otorrinolaringológico y neuroquirúrgico) permitió una resolución favorable. Cabe destacar que cualquier elemento clínico que sugiera compromiso neurológico en el transcurso de una sinusitis debería alertarnos en relación a esta complicación.

Agradecimientos: A José Fuenzalida, radiólogo, por la interpretación de las imágenes.

\section{Resumen}

El empiema subdural es una complicación infrecuente de la sinusitis en niños. Esta condición se acompaña de una alta morbi mortalidad, por lo que es considerada una emergencia neuro-quirúrgica. Se describe la evolución clínico imagenológica de un escolar previamente sano que cursa con un empiema subdural secundario a una pansinusitis y se discute la presentación clínica, diagnóstico imagenológico y tratamiento médico-quirúrgico de esta patología en base a una revisión de la literatura.

\section{Bibliografía}

1.- Bait Merrit M, Shah S, Zaouthis T, Bell L, Feudtner C. Suppurative intracranial complication of sinusitis in previously healthy children. Pediatr Infect Dis J: 2005; 24: 384-6.

2.- Tummala R, Chu R, Hall W. Subdural empyema in children. Neurosurg Q 2004; 14: 257-66.

3.- Bockova J, Rigamonti D. Intracranial empyema. Pediatr Infect Dis J 2000; 19 : 735-7

4.- Lerner ND, Chois S, Zalzal HG, Johnson DL.
Intracranial complications of sinusitis in childhood. .Ann Otol Rhinol Laryngol 1995; 104: 288-93.

5.- Nathoo N, Sameer N, Rikus van Dellen J, Gouws E. Intracranial subdural empyemas in the era of computed tomography: A review of 699 Cases. Neurourgery 1999; 44: 529-36.

6.- Skelton R, Maixner W, Isaacs D Sinusitis induced subdural empyema. Arch Dis Child 1992; 67: 1478-80.

7.- Komori H, Takagishi T, Okati e et al . The efficacy of MR imaging in subdural empyema. Brain Dev 1992; 14: 123-5.

8.- Caro D G, Riedel K I, García C P. Caracteri- zación clínica y microbiológica de las infecciones causadas por Streptococcus grupo anginosus. Rev Chil Infect 2004; 21 : 254-60.

9.- Hoyt DS, Samuel RF: Otolaryngologic management of patients with subdural empyema. Laryngoscope 1991; 101: 20-4.

10.- Rosenfeld EA, Rowley AH. Infectious intracranial complications of sinusitis, other than meningitis in Children: 12 year Review. Clin Infect Dis 1994; 18: 750-4.

11.- Reeves Dill S, Cobbs GC, Mc Donald CK. Subdural empyema: analyses of 32 cases and review. Clin Infect Dis 1995; 20: 372-86. 